\title{
Molecular epidemiology of enteroviruses and predominance of echovirus 30 in an Iranian population with aseptic meningitis
}

\author{
Fatemeh Farshadpour ${ }^{1,2}$ (D) $\cdot$ Reza Taherkhani ${ }^{1,2}$ (])
}

Received: 14 November 2020 / Revised: 27 February 2021 / Accepted: 17 March 2021 / Published online: 31 March 2021

(c) Journal of NeuroVirology, Inc. 2021

\begin{abstract}
Human enteroviruses are the most prevalent causes of aseptic meningitis worldwide. However, despite such predominancy, defining the enteroviral etiology of aseptic meningitis remains a diagnostic dilemma for the clinician in Iran. Therefore, this study was conducted to characterize the prevalence and clinical significance of enteroviral aseptic meningitis as well as the predominant enterovirus serotypes among patients with aseptic meningitis in the South of Iran.

Cerebrospinal fluid (CSF) specimens were obtained from 73 patients with aseptic meningitis $(52.1 \%$ males and $47.9 \%$ females), ages ranging from 1 month to 88 years. Following the extraction of nucleic acid, the detection of enteroviruses was performed by RT-PCR, targeting the $5^{\prime}$ untranslated region of the genome, and sequencing. Enteroviruses were found in $46.6 \%$ of samples (34/73). The most predominant serotype was echovirus 30, followed by coxsackievirus B5 and poliovirus type 1 Sabin strain. The enterovirus infections were more prevalent among female patients (58.8\%) and those below 5 years of age (52.9\%). Although enterovirus infections were observed throughout the year, the infections were more prevalent during autumn with fever as the predominant clinical symptom. The outcomes revealed that enteroviruses are significant causes of aseptic meningitis in the South of Iran, while suspected cases of aseptic meningitis are usually monitored by bacterial culture and biochemical testing of CSF samples. Therefore, the etiology remains unknown in most cases. Molecular detection of viral pathogens should be included as a common approach in the screening of patients with aseptic meningitis to prevent unnecessary treatment and to improve clinical management.
\end{abstract}

Keywords Aseptic meningitis $\cdot$ Enteroviruses $\cdot$ Prevalence $\cdot$ Iran

\section{Introduction}

Enteroviruses are members of the family Picornaviridae. There are four species (A, B, C, and D) and over 100 distinct serotypes of enteroviruses capable of causing human infections (Kumar et al. 2013; Zhu et al. 2016). These nonenveloped positive-stranded RNA viruses are predominantly acquired through the fecal-oral route; however, transmission through respiratory droplets is also possible (Rudolph et al. 2016). The clinical manifestations of human enteroviruses vary from asymptomatic to minor febrile illness or even

Reza Taherkhani

r.taherkhani@bpums.ac.ir

1 Department of Virology, School of Medicine, Bushehr University of Medical Sciences, Moallem Street, 7514633341 Bushehr, Iran

2 Persian Gulf Tropical Medicine Research Center, Bushehr University of Medical Sciences, Bushehr, Iran severe diseases of the central nervous system such as acute flaccid paralysis, encephalitis, and aseptic meningitis (Chen et al. 2018; Tao et al. 2014).

Aseptic meningitis is usually an acute self-limiting infectious disease with meningeal symptoms such as headache, fever, photophobia, vomiting, nausea, and neck stiffness, although the symptoms are not specific and cannot distinguish bacterial from viral meningitis. Additionally, the bacterial culture of cerebrospinal fluid (CSF) is negative with CSF pleocytosis. It usually affects all ages, though younger ages are more vulnerable (Kumar 2005; Rudolph et al. 2016; Wright et al. 2019).

Human enteroviruses are the most prevalent causes of viral meningitis worldwide (Chen et al. 2018; Holmes et al. 2016; Shaker and Abdelhamid 2015; Toczylowski et al. 2019). Nevertheless, the molecular diagnosis of enteroviral meningitis is not a common approach in the public health center of Iran, and diagnosis is based on CSF analysis and clinical findings. While these approaches are incapable of 
defining the etiology of viral meningitis, therefore, the exact etiology remains unknown. These circumstances result in unnecessary administration of antimicrobial agents and prolonged hospitalization and medical expenses. Therefore, this study was conducted to characterize the prevalence, clinical significance, time distribution, and age distribution of enteroviral aseptic meningitis as well as the predominant enterovirus types among patients with aseptic meningitis residents in the northern shores of the Persian Gulf, South of Iran. This is the first report on the molecular epidemiology of enteroviruses among patients with aseptic meningitis in this territory.

\section{Subjects and methods}

\section{Patients and sample collection}

This study was funded by Bushehr University of Medical Sciences with grant number 4359. The patients or their legal guardians were requested to sign a written informed consent to use their leftover CSF samples before enrolment. CSF specimens were obtained from 73 patients with aseptic meningitis who were admitted to the hospitals of Bushehr University of Medical Sciences. Demographic information, clinical data, and informed consent at the time of CSF collection were obtained. In accordance with the diagnostic criteria of aseptic meningitis, aseptic meningitis cases were defined as patients with symptoms and signs suggestive of meningitis, including severe frontal headache, high-grade fever (>38 C), drowsiness, generalized weakness, nuchal rigidity, vertigo, nausea, vomiting or irritability: accompanied by negative CSF bacterial culture, CSF pleocytosis (WBCs $\geq 5$ cells $/ \mathrm{mm}^{3}$ with the predominance of lymphocytes), normal glucose levels ( $>45 \mathrm{mg} / \mathrm{dL}$ ) and increased protein concentrations of CSF (Chadwick 2005; Kaur and Perera 2020; Kumar 2005; Vidal et al. 2011; Wright et al. 2019). Patients were excluded from the study if they had symptoms and signs of brain parenchymal involvement or encephalitis such as obtundation, seizure, or epileptic spasm as well as the positive bacterial culture of CSF or CSF biochemical and cytological pattern of bacterial meningitis. This study was approved by the Ethical Committee of Bushehr University of Medical Sciences with reference number bpums.rec.1394.29.

\section{Molecular detection of enteroviruses}

The viral RNA was extracted from the CSF samples using High Pure Viral Nucleic Acid kit (Roche, Mannheim, Germany) according to the manufacturer's instructions. Following the extraction of nucleic acid, the detection of enteroviruses was performed by nested reverse transcriptase-polymerase chain reaction (nested RT-PCR) assay, targeting the $5^{\prime}$ untranslated region of the genome, and sequencing. First, the viral RNA was reverse-transcribed into cDNA using the SuperScript III cDNA synthesis kit (Invitrogen, Carlsbad, CA, USA). Then, cDNA was amplified by semi-nested RT-PCR using outer primers [forward primer (EV06-F1): CAAGCACTTCTGTTTCCCCGG; reverse primer (EV03-R1): ATTGTCACCATAAGCAGCCA] and inner primers [forward primer (EV06-F1): CAAGCACTT CTGTTTCCCCGG; reverse primer (EV05-R2): CACGGA CACCCAAAGTA] (Boxman et al. 2006; Hsu et al. 2008). The amplified 438 bp length fragment was sequenced to determine the serotypes of enteroviruses (Macrogen Co., Korea). The obtained sequences were used for serotype identification by using GenBank Basic Local Alignment Search Tool (BLAST). The enterovirus sequences isolated from aseptic meningitis patients were submitted to the GenBank sequence database under the accession numbers. These sequences and the nucleotide sequences of enterovirus strains available at the nucleotide database of the NCBI were aligned by ClustalW program in MEGA software version 6.0 (Biodesign Institute, Tempe, AZ, USA). The phylogenetic tree was constructed by the neighbor-joining method using MEGA 6 software, as described previously (Taherkhani et al. 2015).

\section{Statistical analysis}

Data were presented as means $\pm \mathrm{SD}$, frequencies, and percentages following analysis by descriptive statistics. Data were compared between enteroviruses-positive and enteroviruses-negative aseptic meningitis patients using the Student's $t$ test and the $\chi_{2}$ test or the Fisher exact test, when appropriate, and $p$ values $<0.05$ were considered statistically significant. To determine the variables associated with the prevalence of enteroviruses among aseptic meningitis patients, the odds ratio (OR) with $95 \%$ confidence intervals (CI) was computed by logistic regression analysis. All statistical calculations were performed using SPSS 17 package program (SPSS Inc., Chicago, IL, USA).

\section{Results}

CSF specimen were obtained from 73 patients with aseptic meningitis (52.1\% males and $47.9 \%$ females), ages ranging from 1 month to 88 years (median age, 13 years). Of these, 27 patients (37.0\%) were infants less than 1 year, $14(19.2 \%)$ were $1-5$ years old, and the rest were over 5 years old. The highest incidence of aseptic meningitis was reported in autumn (31.5\%) and spring (26.0\%). The mean \pm SD (range) levels of CSF glucose and CSF protein were $61.9 \pm 28.9 \mathrm{mg} /$ $\mathrm{dL}(16-183)$ and $61.1 \pm 48.6 \mathrm{mg} / \mathrm{dL}(10-278)$, respectively. 
Table 1 Prevalence of enterovirus infection according to demographic and quantitative variables among aseptic meningitis in South of Iran

$\begin{array}{llll}\text { No. of all partici- } & \text { No. of PCR Enterovirus posi- } & \text { No. of PCR Enterovirus nega- } & \text { Adjusted OR (95\% CI) }\end{array} \quad P$ value pants (\%): $73(100 \%) \quad$ tive subjects (\%): $34(46.6 \%) \quad$ tive subjects (\%): $39(53.4 \%)$

Age groups (years)

$<1$

1-5

6-14

15-30

31-60

$>60$

Gender

Male

Female

Season

Winter

Spring

Summer

Autumn

Month

Jan

Feb

Mar

Apr

May

Jun

Jul

Aug

Sep

Oct

Nov

Dec

CSF glucose (mg/dL)

$<45$

45-85

$>85$

CSF protein $(\mathrm{mg} / \mathrm{dL})$

$<15$

15-50

$>50$

Lymphocyte \% in CSF

$<30 \%$

30-49\%

$50-74 \%$

$>74 \%$

Antibiotic therapy

Yes

No

Clinical signs

\section{Fever}

Headache

Rash

Drowsiness

$27(37.0 \%)$
$14(19.2 \%)$
$2(2.7 \%)$
$9(12.3 \%)$
$13(17.8 \%)$
$8(11.0 \%)$

$38(52.1 \%)$

$35(47.9 \%)$

$15(20.5 \%)$

19(26.0\%)

$16(21.9 \%)$

23(31.5\%)

$5(6.8 \%)$

$7(9.6 \%)$

$3(4.1 \%)$

$3(4.1 \%)$

$10(13.7 \%)$

$6(8.2 \%)$

$6(8.2 \%)$

$6(8.2 \%)$

$4(5.5 \%)$

$7(9.6 \%)$

$6(8.2 \%)$

$10(13.7 \%)$

$20(27.4 \%)$

$39(53.4 \%)$

$14(19.2 \%)$

$7(9.6 \%)$

$33(45.2 \%)$

$33(45.2 \%)$

$5(6.8 \%)$

$20(38.4 \%)$

$14(8.2 \%)$

$34(46.6 \%)$

$67(91.8 \%)$

$6(8.2 \%)$

$54(74.0 \%)$

$18(24.7 \%)$

$6(8.3 \%)$

$32(43.8 \%)$

$$
\begin{aligned}
& 10(29.4 \%) \\
& 8(23.5 \%) \\
& 1(2.9 \%) \\
& 4(11.8 \%) \\
& 9(26.5 \%) \\
& 2(5.9 \%)
\end{aligned}
$$

$14(41.2 \%)$

$20(58.8 \%)$

$7(20.6 \%)$

$8(23.5 \%)$

$8(23.5 \%)$

11(32.4\%)

$3(8.8 \%)$

$3(8.8 \%)$

$1(2.9 \%)$

2(5.9\%)

$4(11.8 \%)$

2(5.9\%)

1(2.9\%)

$5(14.7 \%)$

2(5.9\%)

2(5.9\%)

4(11.8\%)

$5(14.7 \%)$

$10(29.4 \%)$

$15(44.1 \%)$

$9(26.5 \%)$

$3(8.8 \%)$

$14(41.2 \%)$

$17(50.0 \%)$

$2(5.9 \%)$

$12(35.3 \%)$

$6(17.6 \%)$

$14(41.2 \%)$

$32(94.1 \%)$

$2(5.9 \%)$

$28(82.4 \%)$

$14(41.2 \%)$

$2(5.9 \%)$

$14(41.2 \%)$
$17(43.6 \%)$
$6(15.4 \%)$
$1(2.6 \%)$
$5(12.8 \%)$
$4(10.3 \%)$
$6(15.4 \%)$
$24(61.5 \%)$
$15(38.5 \%)$

$8(20.5 \%)$

11(28.2\%)

$8(20.5 \%)$

$12(30.8 \%)$

$2(5.1 \%)$

4(10.3\%)

2(5.1\%)

$1(2.6 \%)$

$6(15.4 \%)$

4(10.3\%)

$5(12.8 \%)$

$1(2.6 \%)$

2(5.1\%)

$5(12.8 \%)$

2(5.1\%)

$5(12.8 \%)$

$10(25.6 \%)$

$24(61.5 \%)$

$5(12.8 \%)$

$4(10.3 \%)$

$19(48.7 \%)$

$16(41.0 \%)$

$3(7.7 \%)$

$8(20.5 \%)$

$8(20.5 \%)$

$20(51.3 \%)$

$35(89.7 \%)$

$4(10.3 \%)$

$26(66.7 \%)$

$4(10.3 \%)$

$4(10.3 \%)$

$18(46.2 \%)$
1.0

$0.44(0.12-1.64) \quad 0.22$

$0.59(0.03-10.48) \quad 0.72$

$0.73(0.16-1.39) \quad 0.69$

$0.26(0.06-1.07) \quad 0.06$

$1.76(0.3-10.47) \quad 0.53$

1.0

$0.44(0.17-1.12) \quad 0.08$

1.0

$1.2(0.31-4.7) \quad 0.790$

$0.87(0.21-3.59) \quad 0.853$

$0.95(0.26-3.51) \quad 0.944$

1.0

$2.00(0.19-20.61) \quad 0.56$

$3.00(0.15-59.89) \quad 0.47$

$0.75(0.03-14.97) \quad 0.85$

$2.25(0.25-20.13) \quad 0.47$

$3.00(0.25-35.33) \quad 0.38$

$7.50(0.46-122.7) \quad 0.16$

$0.30(0.02-4.90) \quad 0.4$

$1.50(0.11-21.31) \quad 0.76$

$3.75(0.33-42.46) \quad 0.29$

$0.75(0.06-8.83) \quad 0.82$

$1.50(0.17-13.22) \quad 0.71$

1.0

$1.6(0.54-4.75) \quad 0.4$

$0.56(0.14-2.26)$

0.41

1.0

$1.02(0.2-5.3) \quad 0.98$

$0.71(0.14-3.66) \quad 0.68$

NS

NS

NS

CSF cerebrospinal fluid, NS not significant 
Table 2 Comparisons between enterovirus-positive and enterovirus-negative aseptic meningitis patients according to biochemical measurements and duration of hospitalization

\begin{tabular}{llll}
\hline & $\begin{array}{l}\text { Means } \pm \text { SD of all partici- } \\
\text { pants (73) }\end{array}$ & $\begin{array}{l}\text { Means } \pm \text { SD of enterovirus } \\
\text { positive subjects (34) }\end{array}$ & $\begin{array}{l}\text { Means } \pm \text { SD of enterovirus } \\
\text { negative subjects (39) }\end{array}$ \\
\hline CSF glucose (mg/dL) & $61.9 \pm 28.9(16-183)$ & $63.9 \pm 30.9(16-131)$ & $60.1 \pm 27.4(18-183)$ \\
CSF protein (mg/dL) & $61.1 \pm 48.6(10-278)$ & $67.3 \pm 53.4(10-278)$ & $55.8 \pm 44.0(10-168)$ \\
Duration of hospitalization (day) & $10.6 \pm 8.3(1-28)$ & $11.1 \pm 8.5(1-27)$ & $10.1 \pm 8.2(1-28)$ \\
Leukocyte count $/ \mathrm{mm}^{3}$ in CSF & $267 \pm 320(5-1010)$ & $281 \pm 345(5-1010)$ & $230 \pm 261(7-600)$ \\
\hline
\end{tabular}

CSF cerebrospinal fluid

The mean $\pm \mathrm{SD}$ (range) of leukocyte count $/ \mathrm{mm}^{3}$ in CSF was $267 \pm 320 / \mathrm{mm}^{3}\left(5-1010 / \mathrm{mm}^{3}\right)$ with the predominance of lymphocytes in the majority of patients. The most common clinical symptoms were fever, drowsiness, headache, and rash $(74.0 \%, 43.8 \%, 24.7 \%$, and $8.3 \%$, respectively). Antibiotic therapy with empiric antibiotics was reported in $91.8 \%$ of patients. Vancomycin and ceftriaxone antibiotics were administered alone or in combination with other antibiotics in $66 \%$ and $57 \%$ of the patients, respectively. The duration of hospitalization was 1-28 days with the mean \pm SD of 10.6 \pm 8.3 days (Tables 1 and 2).

Of the 73 CSF samples, 34 (46.6\%) were positive for enteroviruses by semi-nested RT-PCR targeting 5'UTR. The enterovirus infections were more prevalent among female patients $(58.8 \%)$ and those below 5 years of age $(52.9 \%)$. Although enterovirus infections were observed throughout the year, the infections were more prevalent during autumn $(32.4 \%, 11 / 34)$. The most predominant clinical symptom was fever, followed by headache and drowsiness. Overall, there were no significant differences between enterovirus-positive and enterovirus-negative aseptic meningitis patients regarding age distribution, gender distribution, the seasonal pattern, the CSF levels of glucose and protein, CSF pleocytosis, and clinical sign and duration of hospitalization (Tables 1 and 2).

Regarding the presence of enterovirus RNA in the CSF samples, 19 samples were found to be positive in the first round of PCR (Fig. 1), and another 15 samples were positive in the second round of PCR (Fig. 2). Out of 19 enteroviruspositive CSF samples, 12 were randomly typed by sequencing (GenBank accession Nos. KX011399-KX011410) (Table 3). The enterovirus sequences isolated from aseptic meningitis patients were identified as echovirus $30(n=8)$, coxsackievirus B5 $(n=2)$, and poliovirus type 1 Sabin strain $(n=2)$, from which echovirus 30 was the predominant serotype (66.6\%) (Fig. 3). These serotypes belonged to human enterovirus $\mathrm{B}$ and $\mathrm{C}$ species.

\section{Discussion}

Viral meningitis, with an estimated incidence of 12 to 19 cases per 100,000 population per year, has been found to be a notifiable infectious disease (Rudolph et al. 2016). Nevertheless, there are few data on the etiology of aseptic meningitis in Iran. Besides, no report is available on molecular identification and clinical characteristics of aseptic meningitis in the northern shores of the Persian Gulf, Iran. Therefore, in this study, we investigated the prevalence and epidemiological pattern of enterovirus infection among aseptic meningitis patients in this territory and found that the prevalence of enterovirus infection was $46.6 \%$, with the predominance of echovirus 30.

The prevalence of enterovirus infections reported in this study is higher than those reported among aseptic meningitis patients in some parts of Iran, 12.5\% in Gorgan (Cheraghali et al. 2013),
Fig. 1 The first round of RTPCR amplification of enterovirus RNA extracted from CSF samples of aseptic meningitis patients by EV06 and EV03 primers. M, 100-bp DNA ladder; $\mathrm{N}$, negative control; $\mathrm{P}$, positive control; $1,2,5,7,8$, and 10 , amplified product $(\approx 438 \mathrm{bp})$ on $2 \%$ agarose gel electrophoresis

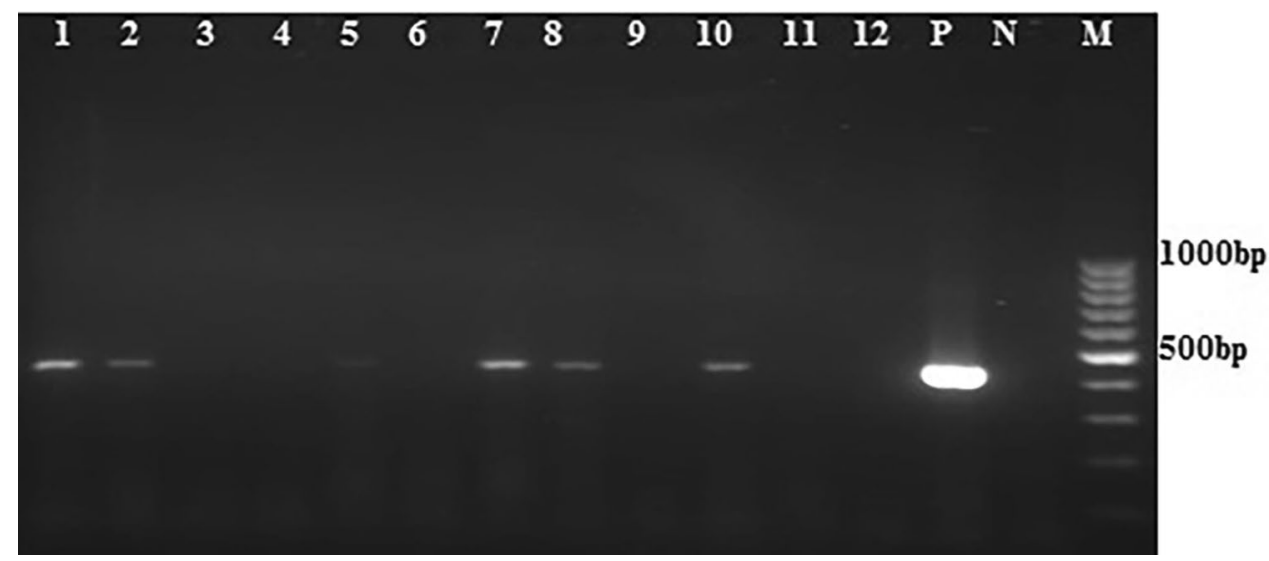




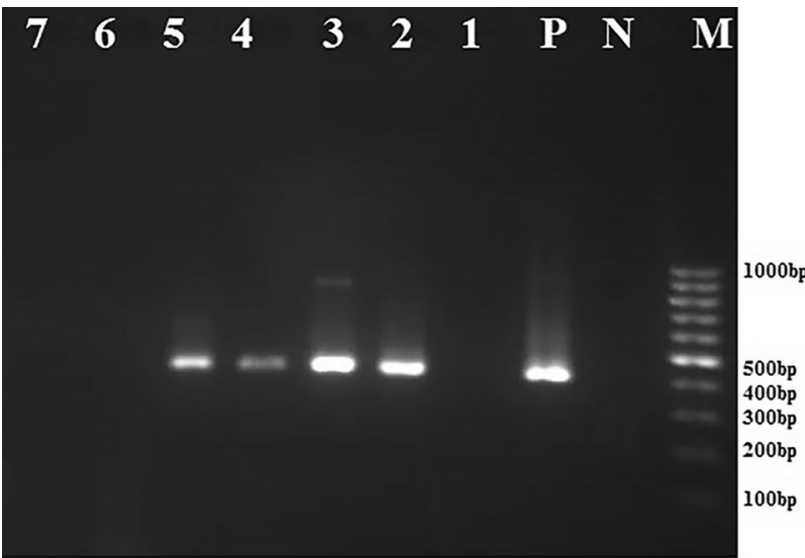

Fig. 2 The second set of semi-nested RT-PCR amplification of negative PCR products from first-round PCR by EV06 and EV05 primers. M, 100-bp DNA ladder; N, negative control; P, positive control; 2, 3, 4 , and 5 , amplified product $(\approx 400 \mathrm{bp}$ ) on $2 \%$ agarose gel electrophoresis

$20 \%$ in Shiraz (Hosseininasab et al. 2011), and 36.2\% in Mashhad (Ghabouli Shahroodi et al. 2016) but lower than those reported among aseptic meningitis patients in Yasuj (51\%) (Moghadam et al. 2020) and Ahvaz (59.6\%) (Rasti et al. 2012). The prevalence of enterovirus infection observed in this study is also higher than those reported among aseptic meningitis patients of some other countries, $16.2 \%$ in Yunnan province, China (Zhu et al. 2016), 18.5\% in Palestine (Dumaidi and Al-Jawabreh 2017), 19.2\% in Shandong province, China (Tao et al. 2014), 24\% in Kuwait (Dalwai et al. 2010), 38.4\% in South Korea (Han et al. 2016), and $43.4 \%$ in Paris-France (Jarrin et al. 2016) but lower than those reported among aseptic meningitis patients in Greece (48.9\%) (Michos et al. 2007), Canada (54.3\%) (Lee et al. 2006), Brazil (83\%) (Vidal et al. 2011), and Poland (67.8\%) (Wieczorek et al. 2016). This variation in the prevalence of enteroviral meningitis may be due to differences in the burden of enterovirus infection in the community, the level of public hygiene and the risk of exposure to enteroviruses in different geographical regions. However, a part of this variation may be due to differences in the sensitivity of molecular assays, sociodemographic characteristics of study population, sample size, and study period in different studies.

In the study of Hosseininasab et al., in Shiraz, Iran, which was performed on 65 patients with suspected aseptic meningitis, contrary to our study, a low prevalence of enterovirus infection (20\%) was reported. In the above study, one-step RT-PCR assay with a commercial kit was performed that was able to detect non-polio enteroviruses (Hosseininasab et al. 2011). While in our study, semi-nested RT-PCR technique was used, which had a very high sensitivity, so that $26 \%$ of enteroviruses were detected in the first round of PCR, and about $20 \%$ of them were found in the second round of PCR. On the other hand, in our study, primers were designed from 5' UTR of the enterovirus genome. In this non-coding region, there are highly conserved nucleotide sequences, so that by designing primers from this region, a wide range of enteroviruses (polio and non-polioviruses) can be detected (Hyypiä et al. 1989; Rotbart 1990). Another difference between our study and the above study is the method of nucleic acid purification. In our study, to obtain the highest sensitivity, nucleic acid extraction was performed by a commercial kit (High Pure Viral Nucleic Acid Kit-Roche, Mannheim, Germany). The main advantage of this kit over the manual method is the simultaneous purification of both viral DNA and RNA and the effective removal of PCR inhibitors in a short time $(\operatorname{Read} 2001)$.

In our study, $52.9 \%$ of enterovirus-positive cases were found among aseptic meningitis patients below 5 years of age. Similarly, in a study in Kuwait, enterovirus infections were more prevalent in the age group under 4 years (Dalwai et al. 2010). In a study in Palestine, $64 \%$ of enterovirus-positive cases were infants ( $<1$ year) (Dumaidi and Al-Jawabreh 2017). In contrast, in a study in Brazil, $81 \%$ of enterovirus-positive cases were in suspected aseptic meningitis patients over the age of 15 years (Bastos et al. 2014). In another study in Spain, 75.2\%
Table 3 Distribution of enterovirus serotypes isolated from aseptic meningitis in southern Iran

\begin{tabular}{lllll}
\hline Accession number & Serotype & Species & Gender & Age \\
\hline KX011399 & Poliovirus type 1 & Human enterovirus C & Male & 25 years \\
KX011400 & Poliovirus type 1 & Human enterovirus C & Female & 17 months \\
KX011401 & Coxsackievirus B5 & Human enterovirus B & Female & 2 months \\
KX011402 & Coxsackievirus B5 & Human enterovirus B & Female & 1 month \\
KX011403 & Echovirus 30 & Human enterovirus B & Female & 13 years \\
KX011404 & Echovirus 30 & Human enterovirus B & Female & 10 months \\
KX011405 & Echovirus 30 & Human enterovirus B & Female & 60 years \\
KX011406 & Echovirus 30 & Human enterovirus B & Male & 37 years \\
KX011407 & Echovirus 30 & Human enterovirus B & Female & 15 months \\
KX011408 & Echovirus 30 & Human enterovirus B & Female & 38 years \\
KX011409 & Echovirus 30 & Human enterovirus B & Male & 68 years \\
KX011410 & Echovirus 30 & Human enterovirus B & Male & 28 years \\
\hline
\end{tabular}


Fig. 3 Neighbor-joining phylogenetic tree based on partial sequences of human enteroviruses isolates (green mark) from the CSF samples of 12 patients with aseptic meningitis in South of Iran (KX011399KX011410). Bootstrap resampling strategy and reconstruction were carried out 1000 times to confirm the reliability of the phylogenetic tree

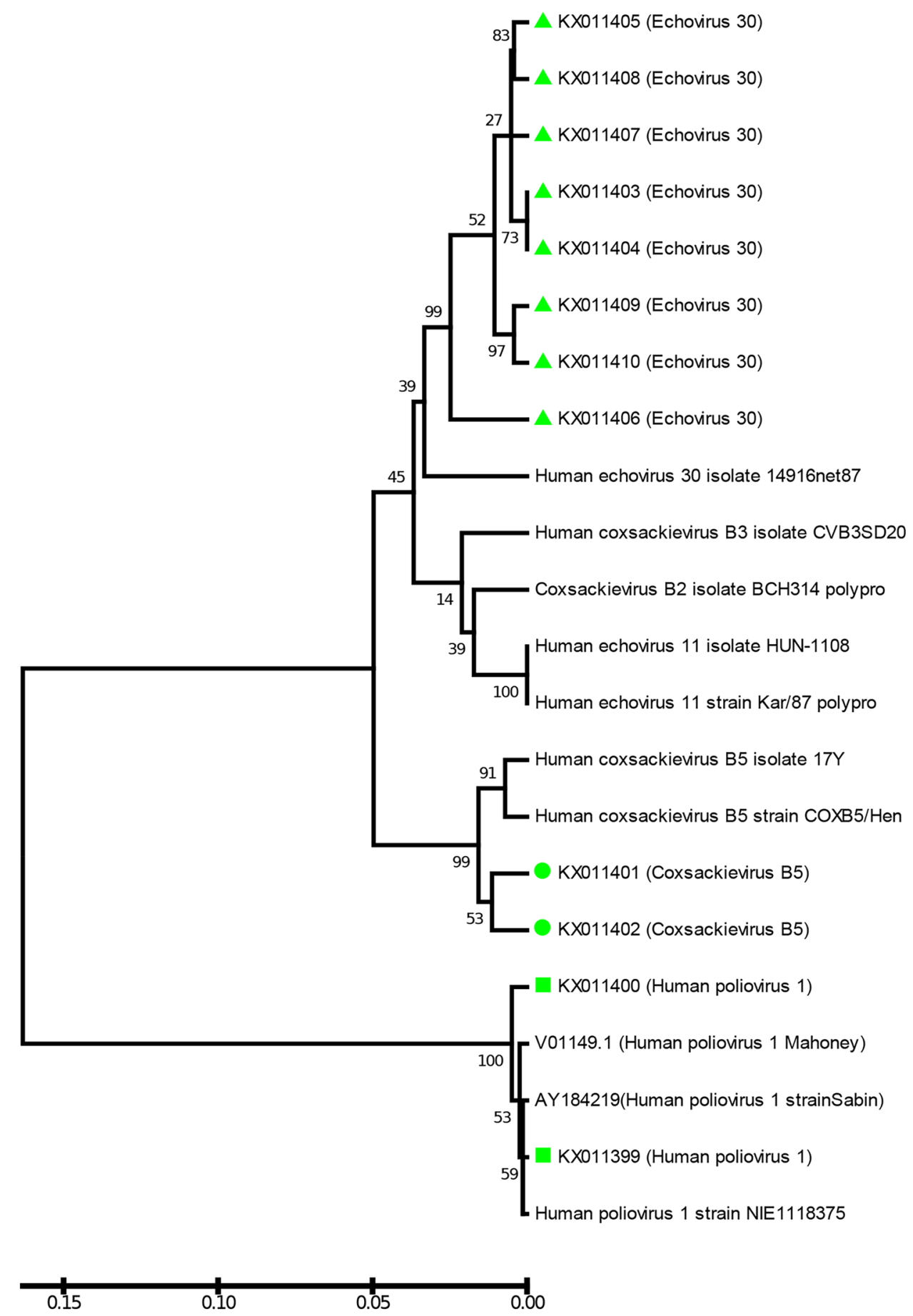

of enterovirus-positive cases were in the age group under 15 years (de Ory et al. 2013). Moreover, in our study, enterovirus infection was more prevalent during autumn. While in Ahwaz (south-west of Iran), most of the enterovirus-positive cases were observed during winter (Rasti et al. 2012). In a study in Poland, the highest rate of enteroviral meningitis was reported in summer and autumn (Wieczorek et al. 2016). In Palestine, enterovirus-positive cases were more prevalent during spring and summer (Dumaidi and Al-Jawabreh 2017).
In the present study, the most predominant serotype was echovirus 30, followed by coxsackievirus B5 and poliovirus type 1 Sabin strain. Similarly, some previous studies have reported high frequencies of echovirus 30 in aseptic meningitis patients in the UK and Shandong province, China (Holmes et al. 2016; Tao et al. 2014). In Kuwait, the most predominant serotype associated with enteroviral meningitis was echovirus 9, followed by echoviruses 11 and 30 (Dalwai et al. 2010). In Palestine, echovirus 6 was the predominant 
serotype (Dumaidi and Al-Jawabreh 2017). In Yunnan province, China, the most predominant serotype was echovirus 9, followed by coxsackievirus B5 and echovirus 30 (Zhu et al. 2016). In another study in Shandong province, China, coxsackievirus B5 was reported as the predominant serotype among viral meningitis patients (Chen et al. 2018). In Poland, the most predominant serotype was echovirus 6 , followed by echovirus 30 (Wieczorek et al. 2016). These differences in the predominance of enterovirus serotypes in different geographical regains indicate various epidemiological patterns of enteroviral meningitis and endemicity of special enterovirus serotypes in different geographical regions.

Although differentiation of enterovirus serotypes has usually less clinical urgency, differentiation of non-polio enteroviruses and polioviruses is important to investigate suspected cases of poliomyelitis, diagnose non-polio enterovirus infection in people who have recently been vaccinated with oral poliovirus vaccine, and monitor the status of circulating wild-type poliovirus in endemic areas (Abraham et al. 1993). Following vaccination against polio and mumps, most cases of aseptic meningitis are caused by non-polio enteroviruses (Chamberlain 2009). While in our study, two cases were positive for poliovirus type 1, including a 25-year-old male and a 17-month-old female. Unfortunately, no information was available on vaccination with oral poliovirus vaccine in their hospital records.

Antiviral therapy is not available for enteroviruses, and treatment is usually supportive, such as controlling fever and relieving headaches (Lyons 2018; Wright et al. 2019). Nevertheless, for enterovirus infections in infants and cases with defective immune systems, intravenous immunoglobulin (IVIG) is prescribed (Yen et al. 2015; Zhang et al. 2015). There is no antiviral drug to treat aseptic meningitis caused by polioviruses, but an effective vaccine is available to prevent it (Chadwick 2005; Taherkhani et al. 2018). The use of molecular assays in the diagnosis of children with enteroviral aseptic meningitis, given that most of them are hospitalized, has a significant impact on reducing the length of hospitalization and the related medical expenses (Steiner et al. 2012; Wright et al. 2019).

\section{Conclusion}

The outcomes revealed that enteroviruses are significant causes of aseptic meningitis in the South of Iran, while suspected cases of aseptic meningitis are usually monitored by bacterial culture and biochemical testing of CSF samples. Therefore, the etiology remains unknown in most cases. The results of our study demonstrate the role of modern microbiological methods such as PCR in the diagnosis of the enteroviral etiology of aseptic meningitis. In addition, the nested
RT-PCR assay increases the sensitivity of detection, so that $44.12 \%$ of positive cases for enteroviruses were detected in the second round of PCR. Altogether, molecular detection of viral pathogens should be included as a common approach in the screening of patients with aseptic meningitis to prevent unnecessary treatment and to improve clinical management.

Acknowledgements The authors would like to acknowledge grant no. 4359 supported by the Deputy Research and Affairs of the Bushehr University of Medical Sciences, Bushehr, Iran.

Author contribution Taherkhani R and Farshadpour F equally contributed to this paper.

Funding This study was funded by Bushehr University of Medical Sciences with grant number 4359.

Data availability All relevant data are within the paper.

\section{Declarations}

Ethical approval This study was approved by the Ethical Committee of Bushehr University of Medical Sciences with reference number bpums. rec.1394.29.

Conflict of interest The authors declare no competing interests.

\section{References}

Abraham R, Chonmaitree T, McCombs J, Prabhakar B, Verde PL, Ogra P (1993) Rapid detection of poliovirus by reverse transcription and polymerase chain amplification: application for differentiation between poliovirus and nonpoliovirus enteroviruses. J Clin Microbiol 31:395-399

Bastos MS, Lessa N, Naveca FG, Monte RL, Braga WS, Figueiredo LTM, Ramasawmy R, Mourao MPG (2014) Detection of Herpesvirus, Enterovirus, and Arbovirus infection in patients with suspected central nervous system viral infection in the Western Brazilian Amazon. J Med Virol 86:1522-1527

Boxman IL, Tilburg JJ, Te Loeke NA, Vennema H, Jonker K, De Boer E, Koopmans M (2006) Detection of noroviruses in shellfish in the Netherlands. Int J Food Microbiol 108:391-396

Chadwick DR (2005) Viral meningitis. Br Med Bull 75:1-14

Chamberlain NR (2009) Meningitis and Encephalitis. In: The Big Picture, Medical Microbiology. The McGraw-Hill Companies: New York, USA, pp 456

Chen P, Lin X, Liu G, Wang S, Song L, Tao Z, Xu A (2018) Analysis of enterovirus types in patients with symptoms of aseptic meningitis in 2014 in Shandong, China. Virology 516:196-201

Cheraghali F, Moradi A, Azadfar S, Javid N, Tabarraei A (2013) Molecular epidemiology of viral meningitis in children in south east of Caspian Sea Iran. Int J Mol Clin Microbiol 1:237-243

Dalwai A, Ahmad S, Al-Nakib W (2010) Echoviruses are a major cause of aseptic meningitis in infants and young children in Kuwait. Virol J 7:236

de Ory F, Avellon A, Echevarria JE, Sanchez-Seco MP, Trallero G, Cabrerizo M, Casas I, Pozo F, Fedele G, Vicente D, Pena MJ, Moreno A, Niubo J, Rabella N, Rubio G, Perez-Ruiz M, Rodriguez-Iglesias M, Gimeno C, Eiros JM, Melon S, Blasco M, Lopez-Miragaya I, Varela E, Martinez-Sapina A, Rodriguez G, Marcos MA, Gegundez MI, 
Cilla G, Gabilondo I, Navarro JM, Torres J, Aznar C, Castellanos A, Guisasola ME, Negredo AI, Tenorio A, Vazquez-Moron S (2013) Viral infections of the central nervous system in Spain: a prospective study. J Med Virol 85:554-562

Dumaidi K, Al-Jawabreh A (2017) Molecular detection and genotyping of enteroviruses from CSF samples of patients with suspected sepsis-like illness and/or aseptic meningitis from 2012 to 2015 in West Bank Palestine. PLoS ONE 12:e0172357

Ghabouli Shahroodi MJ, Ghazvini K, Sadeghi R, Sasan MS (2016) Enteroviral meningitis in neonates and children of Mashhad Iran. Jundishapur J Microbiol 9:e19955

Han SH, Choi HY, Kim JM, Park KR, Youn YC, Shin HW (2016) Etiology of aseptic meningitis and clinical characteristics in immunecompetent adults. J Med Virol 88:175-179

Holmes CW, Koo SS, Osman H, Wilson S, Xerry J, Gallimore CI, Allen DJ, Tang JW (2016) Predominance of enterovirus B and echovirus 30 as cause of viral meningitis in a UK population. J Clin Virol 81:90-93

Hosseininasab A, Alborzi A, Ziyaeyan M, Jamalidoust M, Moeini M, Pouladfar G, Abbasian A, Kadivar MR (2011) Viral etiology of aseptic meningitis among children in southern Iran. J Med Virol 83:884-888

Hsu BM, Chen CH, Wan MT (2008) Prevalence of enteroviruses in hot spring recreation areas of Taiwan. FEMS Immunol Med Microbiol 52:253-259

Hyypiä T, Auvinen P, Maaronen M (1989) Polymerase chain reaction for human picornaviruses. J Gen Virol 70:3261-3268

Jarrin I, Sellier P, Lopes A, Morgand M, Makovec T, Delcey V, Champion K, Simoneau G, Green A, Mouly S (2016) Etiologies and management of aseptic meningitis in patients admitted to an internal medicine department. Medicine 95:e2372

Kaur H, Perera TB (2020) Aseptic Meningitis. In: StatPearls: Treasure Island (FL).

Kumar A, Shukla D, Kumar R, Idris MZ, Jauhari P, Srivastava S, Dhole TN (2013) Molecular identification of enteroviruses associated with aseptic meningitis in children from India. Arch Virol 158:211-215

Kumar R (2005) Aseptic meningitis: diagnosis and management. Indian J Pediatr 72:57-63

Lee BE, Chawla R, Langley JM, Forgie SE, Al-Hosni M, Baerg K, Husain E, Strong J, Robinson JL, Allen U, Law BJ, Dobson S, Davies HD (2006) Paediatric investigators collaborative network on infections in Canada (PICNIC) study of aseptic meningitis. BMC Infect Dis 6:68

Lyons JL (2018) Viral meningitis and encephalitis. Continuum (Minneap Minn) 24:1284-1297

Michos AG, Syriopoulou VP, Hadjichristodoulou C, Daikos GL, Lagona E, Douridas P, Mostrou G, Theodoridou M (2007) Aseptic meningitis in children: analysis of 506 cases. PLoS ONE 2:e674

Moghadam AG, Yousefi E, Ghatie MA, Moghadam AG, Pouladfar GR, Jamalidoust M (2020) Investigating the etiologic agents of aseptic meningitis outbreak in Iranian children. J Family Med Prim Care 9:1573-1577

Rasti M, Samarbaf-Zadeh AR, Makvandi M, Shamsi-Zadeh A (2012) Relative frequency of enteroviruses in children with aseptic meningitis referred to Aboozar Hospital in Ahvaz. Jundishapur J Microbiol 5:456-459
Read S (2001) Recovery efficiencies of nucleic acid extraction kits as measured by quantitative Light Cycler TM PCR. Mol Pathol 54:86

Rotbart HA (1990) Enzymatic RNA amplification of the enteroviruses. J Clin Microbiol 28:438-442

Rudolph H, Schroten H, Tenenbaum T (2016) Enterovirus infections of the central nervous system in children: an update. Pediatr Infect Dis J 35:567-569

Shaker OG, Abdelhamid N (2015) Detection of enteroviruses in pediatric patients with aseptic meningitis. Clin Neurol Neurosurg 129:67-71

Steiner I, Schmutzhard E, Sellner J, Chaudhuri A, Kennedy P (2012) EFNS-ENS guidelines for the use of PCR technology for the diagnosis of infections of the nervous system. Eur J Neurol 19:1278-1291

Taherkhani R, Farshadpour F, Makvandi M, Hamidifard M, Esmailizadeh M, Ahmadi B, Heidari H (2015) Determination of cytomegalovirus prevalence and glycoprotein B genotypes among ulcerative colitis patients in Ahvaz, Iran. Jundishapur J Microbiol 8:e17458

Taherkhani R, Farshadpour F, Ravanbod MR (2018) Vaccine-associated paralytic poliomyelitis in a patient with acute lymphocytic leukemia. J Neurovirol 24:372-375

Tao Z, Wang H, Li Y, Liu G, Xu A, Lin X, Song L, Ji F, Wang S, Cui N (2014) Molecular epidemiology of human enterovirus associated with aseptic meningitis in Shandong Province, China, 2006-2012. PLoS ONE 9:e89766

Toczylowski K, Wojtkowska M, Sulik A (2019) Enteroviral meningitis reduces CSF concentration of Abeta42, but does not affect markers of parenchymal damage. Eur J Clin Microbiol Infect Dis 38:1443-1447

Vidal LRR, Almeida SMd, Messias-Reason IJd, Nogueira MB, Debur MdC, Pessa LFC, Pereira LA, Rotta I, Takahashi GRdA, Silveira CSd (2011) Enterovirus and herpesviridae family as etiologic agents of lymphomonocytary meningitis, Southern Brazil. Arq Neuropsiquiatr 69:475-481

Wieczorek M, Figas A, Krzysztoszek A (2016) Enteroviruses associated with aseptic meningitis in Poland, 2011-2014. Pol J Microbiol 65:231-235

Wright WF, Pinto CN, Palisoc K, Baghli S (2019) Viral (aseptic) meningitis: a review. J Neurol Sci 398:176-183

Yen MH, Huang YC, Chen MC, Liu CC, Chiu NC, Lien R, Chang LY, Chiu CH, Tsao KC, Lin TY (2015) Effect of intravenous immunoglobulin for neonates with severe enteroviral infections with emphasis on the timing of administration. J Clin Virol 64:92-96

Zhang Y, Moore DD, Nix WA, Oberste MS, Weldon WC (2015) Neutralization of Enterovirus D68 isolated from the 2014 US outbreak by commercial intravenous immune globulin products. J Clin Virol 69:172-175

Zhu Y, Zhou X, Liu J, Xia L, Pan Y, Chen J, Luo N, Yin J, Ma S (2016) Molecular identification of human enteroviruses associated with aseptic meningitis in Yunnan province Southwest China. Springerplus 5:1515

Publisher's Note Springer Nature remains neutral with regard to jurisdictional claims in published maps and institutional affiliations. 